\title{
Calibrating and Testing EUSO-SPB in Flight using a Laser and LEDs on an Aircraft
}

\author{
Malek Mastafa* \\ University of Alabama in Huntsville \\ E-mail: mm0050@uah.edu \\ James Adams \\ University of Alabama in Huntsville \\ E-mail: jha0003@uah.edu

\section{Mark Christl} \\ NASA Marshall Space Flight Center \\ E-mail: mark.christl@nasa.gov \\ Lawrence Wiencke and Johannes Eser \\ Colorado School of Mines \\ E-mail: lwiencke@mines.edu, jeser@mymail.mines.edu
}

\section{For the JEM-EUSO Collaboration}

The EUSO-SPB (EUSO super pressure balloon) experiment is designed to make observations of fluorescence light from Extreme Energy Cosmic Ray (EECR) Extensive Air Showers (EASs) by looking down on the atmosphere from above. The flight lasted for 12 days and 4 hours with a total of more than 30 hours of observations on dark nights. The balloon was launched on April 24, 2017, from Wanaka New Zealand. A navigation system consists of MATLAB GUI software together with tracker beacon and Ham radio was designed to track the location of tracking the instrument's field of view. This navigation system was tested prior to the flight in Huntsville, Al. An airplane carrying calibrated UV light emitting diodes (LEDs) and a UV laser was planned to fly under the balloon on a moonless night when it is at its float altitude. This under flight was to calibrate EUSO-SPB using these light sources to produce EECR-like signals. The laser pulses were to calibrate the trigger efficiency and track reconstruction accuracy of the experiment. The flashers will to determine the sensitivity of EUSO-SPB to fluorescent light near the maxima of EASs. Unfortunately, the balloon flight had to be terminated before there was an opportunity for the under flight. This paper describes preparations for the under flight including the design of tracking system and the calibration light sources that were to be carried on the airplane.

35th International Cosmic Ray Conference - ICRC2017

10-20 July, 2017

Bexco, Busan, Korea

* Speaker. 


\section{Introduction}

EUSO-SPB (EUSO super pressure balloon) is an experiment flown on a super pressure balloon. The flight lasted for 12 days 4 hours for estimated total data collection period of more than 30 hours during moon down darkness. The balloon was launched on April 24, 2017 from Wanaka in New Zealand.

The instrument [1] is an updated version of the EUSO-Balloon instrument flown in Ontario, Canada in 2014. It includes a similar JEM-EUSO PDM (photon detection module with 2304 pixels) [2], as in EUSO-Balloon, and an optical system with two Fresnel lenses that are each one square meter area. They cover a field of view of \pm 6 degrees. EUSO-SPB included a trigger to allow the instrument to detect high energy cosmic ray events[3].

The main scientific objective of EUSO-SPB is the first observation and measurements of Ultra High Energy Cosmic Ray Extensive Air Showers by looking down from near space with a fluorescence detector. EUSO-SPB can also search for UV pulse like signatures from other objects as meteoroids and atmosphere transient luminous events (ATLEs) and it will also measure the slowly varying UV light from airglow and bioluminescence over the ocean. Integration of the instrument was completed at the Colorado School of Mines. Laser and UV LED calibrations were done at the Telescope Array site. The hang test was performed at the Columbia Scientific Balloon Facility (CSBF) in Palestine (Texas) prior to shipment of the payload to Wanaka.

A major component of the EUSO-SPB test was to be the underflight. The purpose of the underflight was to provide an in-flight calibration of the EUSO-SPB instrument to supplement the calibration done in Utah. An aircraft flying under the payload was to use two light sources to simulate the optical signatures of Ultra High Energy Cosmic Ray Air Showers. These light sources are a UV LED system, pointed up, and a UV laser system directed out the side of the aircraft. The UV LED system can simulate vertical air showers and the laser will simulate air shower with incident angles between $0^{\circ}$ and $30^{\circ}$ below the horizontal. Because the intrinsic luminosity of both light sources is known, the EUSO science team can characterise the flying EUSO instrument using the data collected from the underflight.

The underflight was to happen after the balloon made one orbit. A cesna 421C aircraft was identified and instrument installation was in preparation at the time the balloon flight was terminated prematurely. In this paper we will describe all the tools that we had developed for the underflight including, the navigation software, the tracker beacon and the Ham radio. We will also describe the characterisation of the UV light emitting diodes(LEDs) and the UV laser that was supposed to be used during the underflight test.

\section{Pre-flight Preparation}

\subsection{Design and testing of the balloon tracker system}

The main purpose of the tracker system was to locate the balloon from the airplane in real time and then to support navigation of the airplane within the field of view of EUSO-SPB. The parameters that the tracker system needed to provide to assure the underflight mission is successful are the balloon position (included location, compass heading, and altitude) and the airplane position (included location, compass heading, inertial reference frame and altitude). A tracker beacon 
consisting of a GPS receiver coupled with a HAM transmitter was developed in order to transmit the position of the balloon, see Figure 1. This tracker beacon was located on EUSO-SPB.
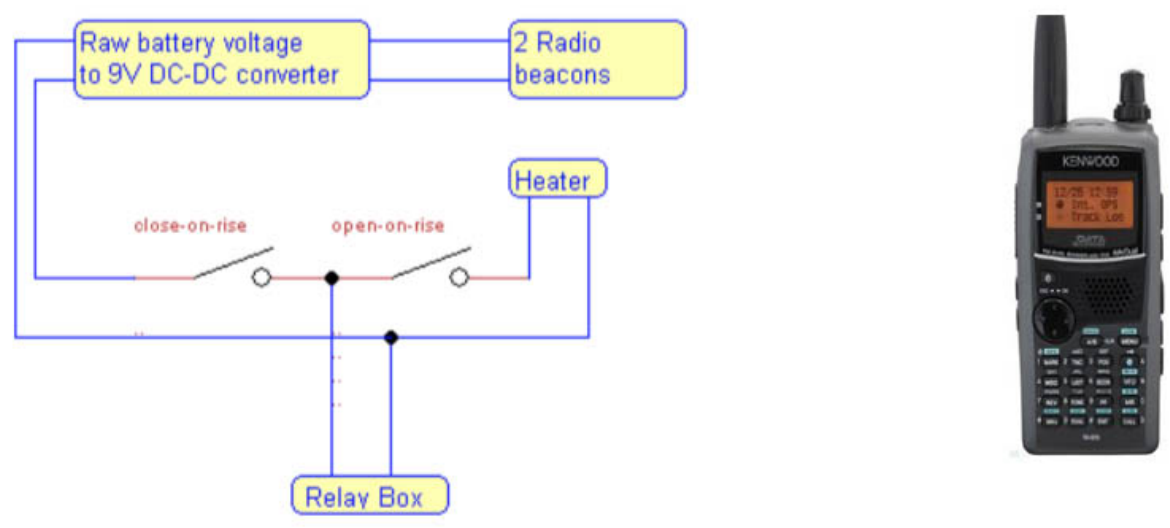

Figure 1: Kenwood ham radio and the schematic drawing of the tracker beacon circuit.

The HAM signals from either two redundant tracker beacons could be received on the airplane using a Kenwood software-defined radio. These radio beacons were turned on during the climb out of the balloon after its launch to confirm that both were working. They were to have been turned on again prior to the underflight. The primary purpose of the trackers is to provide the underflight team with the information required to perform the maneuvers for calibration while under the balloon. These tracker beacons are powered by the $24 \mathrm{~V}$ EUSO-SPB power bus. A heater inside the tracker package is used to warm the trackers after they were powered on until they were in a safe-operatingtemperature. A thermal switch turned on the heater when the temperature was below $-30 \mathrm{C}$ and off when the temperature is above $0 \mathrm{C}$. A thermal switch on the tracker power is set to turn them off when the temperature gets below $-37 \mathrm{C}$ because lower temperatures were outside their operating range. The balloon's avionics has the ability to actuate power to the tracker beacons. The data transmitted by the trackers is based solely on GPS receiver and was to be recorded on the aircraft for later processing. The transmitters send out a data packet once every 5 seconds. The trackers operated on 2 separate frequencies $449.93125 \mathrm{MHz}$ and $449.95625 \mathrm{MHz}$. Data packets from the trackers are APRS-encoded packets. The trackers were both transmitting using the Amateur Radio Callsign of KM4WOI. The communication and RF protocols used by the trackers are 2-GFSK, 9600 Baud using the G3RUH standard.

Onboard the Aircraft Cessna 421C, 2 GPSs were available to the underflight team to collect location, altitude, speed, and compass heading information and log it to a data file on a laptop onboard the aircraft. The primary purpose of these GPSs was to provide real-time data on the aircraft's position to the underflight team to help them perform the manoeuvres under the SPB required for the underflight. The GPS data was to be collected at $1 \mathrm{~Hz}$.

A custom Matlab GUI is developed and used to collect all the underflight GPS data required to perform the navigation. This software was to be able to provide the team of two plots. One showed 
the track of the balloon relative to the airplane with a circle showing the field of view. A second plot showed the track of the airplane relative to that of the balloon. These are shown in Figure 2.

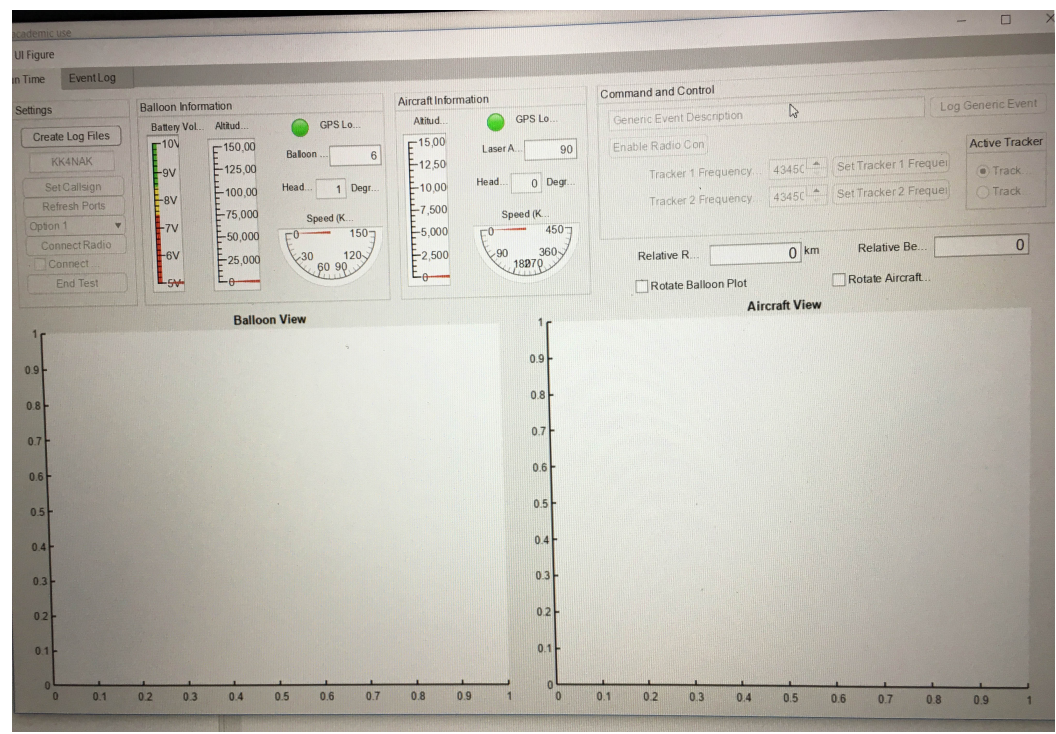

Figure 2: The interface of the custom Matlab GUI

\subsection{Tracker System test}

To test the navigation system performance, a flight test was conducted at Huntsville,AL,USA. During the flight a $1500 \mathrm{~g}$ latex balloon that took off from the tracker beacons was launched by the members of the UAH Space Hardware Club. The balloon was chased by the airplane carrying from the Madison County Municipal Airport some 20 miles away. Two of our team members to operate the custom Matlab GUI and provided the pilot with instructions to navigate the airplane under the balloon.

The aircraft was successfully navigated under the balloon where it was guided to perform a series of maneuvers to practice different scenarios for firing the LED flashers and the UV laser as would be done inside the field of view and maneuvering at the edge of the field of view of EUSO-SPB during the underflight as shown in the Figure 3.

\section{Global Light System}

To calibrate EUSO-SPB, two light sources of known intrinsic luminosity were to be mounted in the baggage compartments in the nose of the Cessna $421 \mathrm{C}$ aircraft. These were a calibrated UV-emiting LED flasher and a UV laser.

The UV-flasher contains 4 LED chips (TH-UV365T4WA-6363 / 365nm) with a total optical power 2.35W [4] (see Figure 4). The UV LEDs were calibrated in the lab at 25 degree C, to verify its calibration it was tested with the EUSO-TA instrument from remote test site within the field of view of EUSO-TA on October 3, 2016. UV-flasher is equipped by current stabilization circuit, which maintains constant current through the LED chips during the applied strobe. The angular 


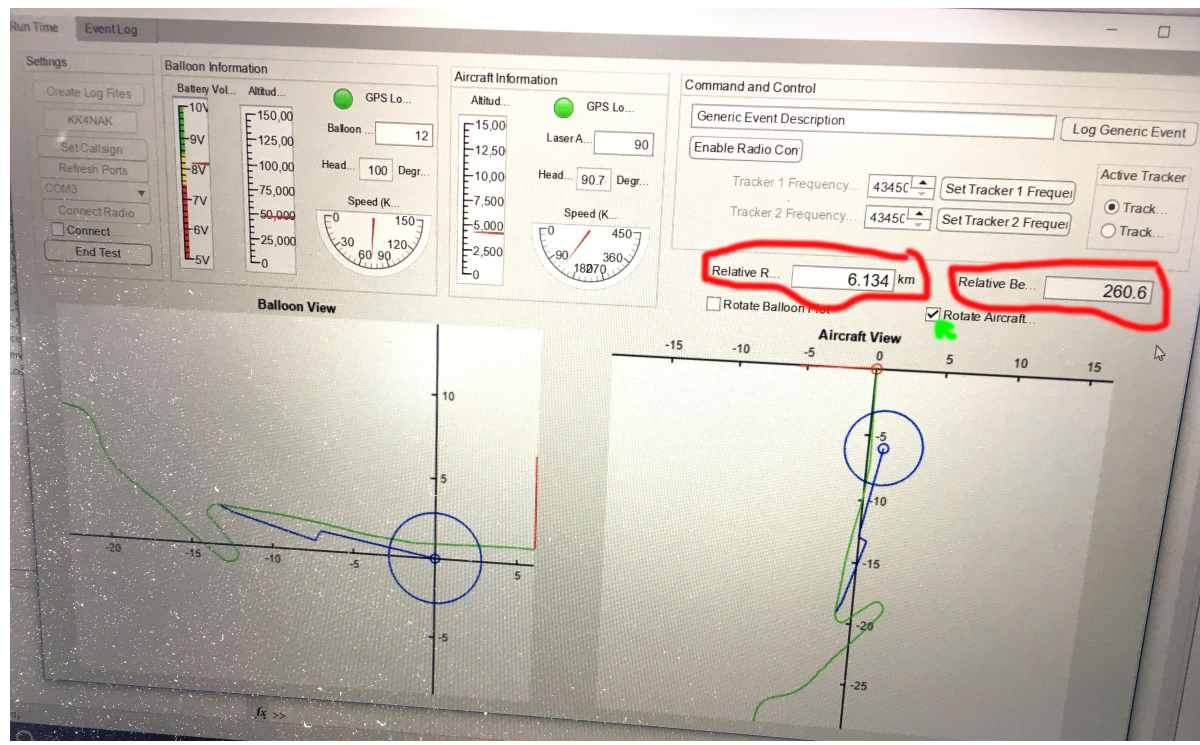

Figure 3: Matlab GUI during the flight, the blue circle is the field of view of the balloon, and the green line is the path of the airplane

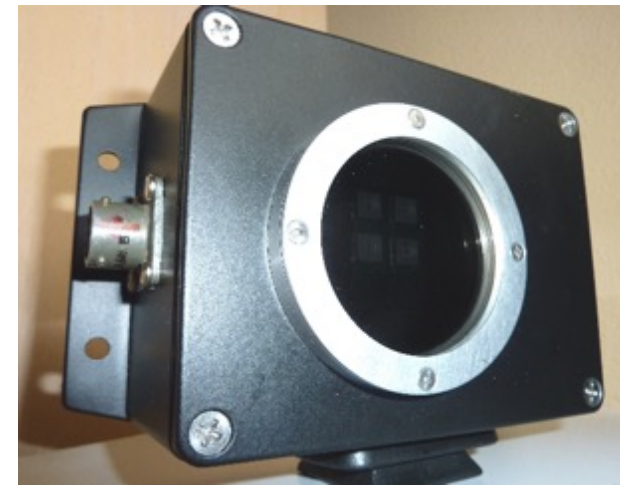

Figure 4: UV Flasher with installed $10 \%$ neutral density filter

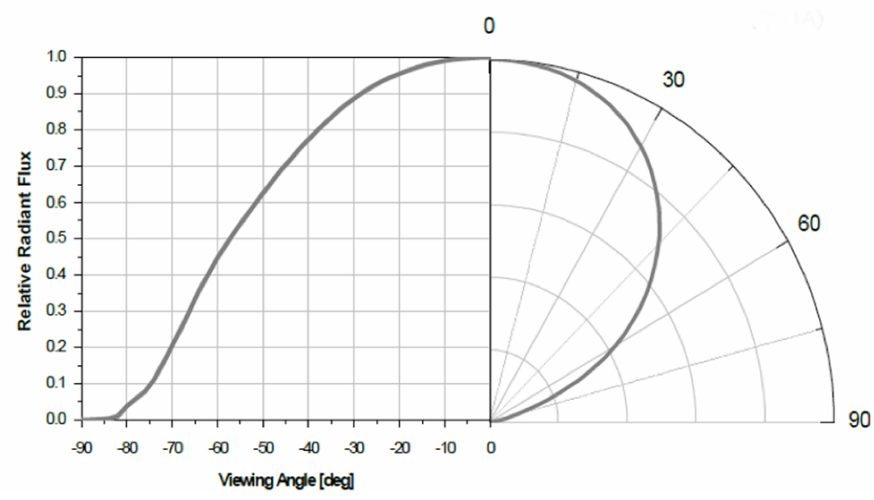

Figure 5: Angular distribution of the LED chip

distribution of a single LED chip is shown in the Figure 5.

The UV laser [5] to be flown is a Quantel CFR-Ultra pulsed YAG-laser with frequency trippling to $355 \mathrm{~nm}$ ( see Figure 6). Its maximum energy pulse is $18 \mathrm{~mJ}$ and the pulse width is $7 \mathrm{~ns}$. All the characteristics of the laser beam are listed in the Table 1 .

These two light sources were supposed to fire as in a sequence(LED flasher and then Laser) from the the airplane when it will reach the field of view of the instrument. The plan was to wait for the balloon to circle the Earth and return back again near New Zealand or Australia in a moonless night and then it would be chased by the airplane. The estimated flight time the underflight was around 3 hours, but since the mission was terminated due to the leak of the helium from the balloon, the underflight was cancelled. 


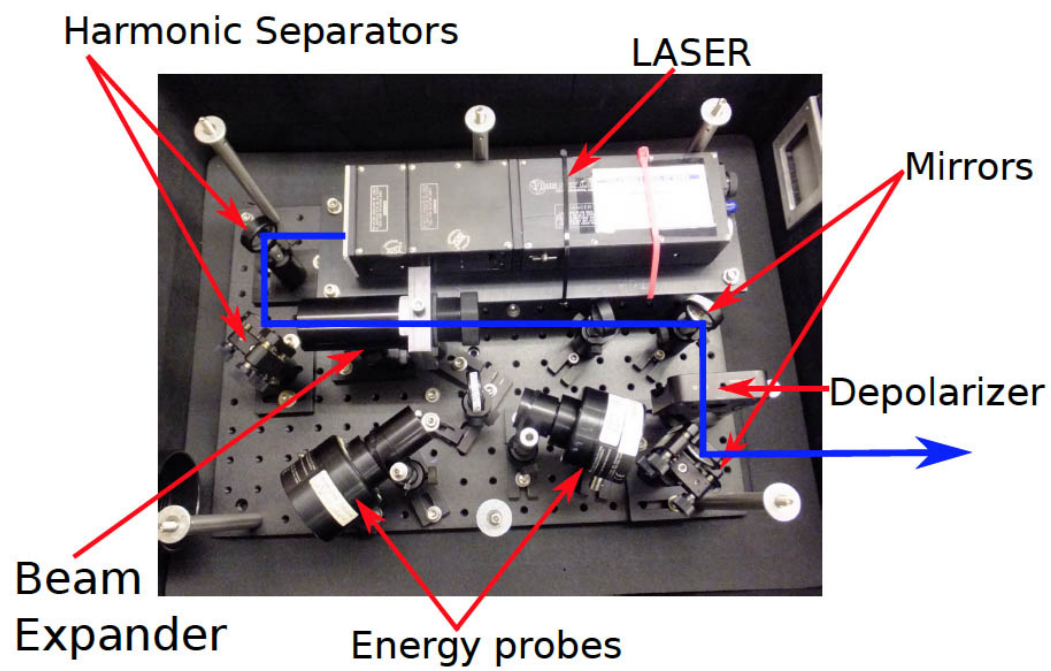

Figure 6: The UV-laser system

\begin{tabular}{lclc}
\hline wavelength & $355 \mathrm{~nm}$ & divergence & $<0.04^{\circ}$ \\
\hline relative energy calibration & $>2 \%$ & beam halo & $<0.5 \%$ \\
\hline absolute energy calibration & $>4.5 \%$ & spectral purity & $>99.9 \%$ \\
\hline $\begin{array}{l}\text { overall stability } \\
\text { depolarization }\end{array}$ & $1.2 \%$ & beam pointing & $\begin{array}{c}\text { vertical: } 3^{\circ} \\
\text { horizontal: } 1^{\circ}\end{array}$ \\
\hline absolute timing accuracy & $\pm 20 \mathrm{~ns}$ & &
\end{tabular}

Table 1: Laser beam characteristics [5]

\section{Underflight Scenarios}

Before performing the underflight, the EUSO team developed several scenarios for what should be the airplane banking angle and its path during the shooting of the laser and the flashers. We assumed several parameters related to the flight (see Table 2).

\begin{tabular}{lcl}
\hline parameter & $\mathrm{ft}$ & meter \\
\hline average altitude of the balloon & 110000 & 33528 \\
average altitude of the airplane & 10000 & 3048 \\
\hline
\end{tabular}

Table 2: The assumed parameters for the flight.

Since the field of view angle is \pm 6 degrees, the radius of the circle of the field of view is 3203 meters as shown in the Figure 7. The banking angle of the airplane (when the airplane is circling at the of edge of the field of view) was such that the flasher needed to emit uniformly out to 25.4 degrees from its optical axis in order to illuminate EUSO-SBP with its calibrated intrinsic luminosity.

In the case of laser the airplane should follow the balloon at the edge of its field of view. When circling the field of view, the airplane will be banking at angle 25.4 degrees to balance the centrifu- 


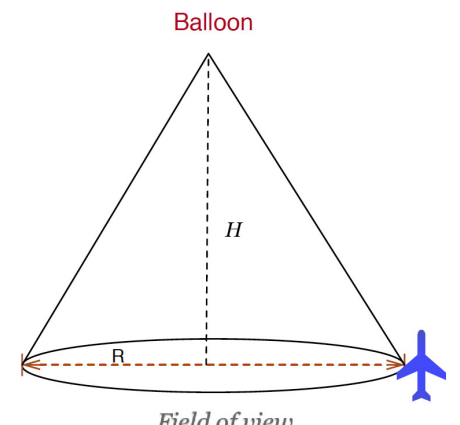

Field of view

\section{ocean}

Figure 7: The circle of the field of view, $\mathrm{H}$ is the relative height between the airplane and the balloon

gal force of the turn as shown in the Figure 7.

In the case of triggering LED flasher, the best way is that the airplane follow the direction of the balloon entering the inside the field of view and then turn on outside the field of view and return for a second pass.

\section{Conclusion}

Following a successful aircraft underflight of the EUSO-Balloon 2014 campaign, we updated a package of lights sources (LEDs and UV lasers), navigation tools including software and trackers for the EUSO-SPB underflight. A Cessna 421C aircraft was in preparation to intercept the balloon after one circumnavigation. Unfortunately the balloon flight was terminated prematurely so no underflight was performed.

\section{Acknowledgment}

Attitude to Matthew Rodencal for his tireless help with the navigation system. We also want to thank our pilot, Jon Bell of the Executive Flight Service Center and the UAH Space Hardware Club who launched the test balloon for us and Nathaniel Stepp for lending assistance with his drone.

\section{References}

[1] S.Bacholle. The EUSO-SPB Instrument, in proceeding of the 35th ICRC, (2017).

[2] J.Adam, et al., JEM-EUSO collaboration, An evaluation of the exposure in nadir observation of the JEM-EUSO mission, Astroparticle Physics 44 (2013): 76-90.

[3] G. Abdellaoui, et al., JEM-EUSO collaboration, Cosmic Ray Oriented Performance Studies for the JEM-EUSO First Level Trigger, Nuclear Instruments and Methods in Physics Research Section A: Accelerators, Spectrometers, Detectors and Associated Equipment 866 (2017): 150-163. . 
[4] E.Kuznetsov and J.Watts. Absolute calibration of the Modified UV Flasher with EUSO-TA instrument, (2016).

[5] J.Eser, et al., JEM-EUSO collaboration, EUSO-balloon: Observation and measurement of Tracks from a laser in a helicopter, 34th ICRC, (The Hague), (2015). 